Revista Colombiana de Ciencia Animal RECIA ISSN: 2027-4297

reciaeditor@unisucre.edu

Universidad de Sucre

Revista Colombiana de Ciencia Animal Colombia

\title{
Descripción radiológica de lesiones en el tarso de caballos criollos colombianos
}

\author{
Radiological description of lesions in the tarsus of colombian creole \\ horses
}

Gallego-Rodríguez RS. Lujan-Giraldo JD. Leysner-Tavera J. Descripción radiológica de lesiones en el tarso de caballos criollos colombianos. Rev Colombiana Cienc Anim. Recia. 2019; 11(2):Articulo728. DOI: https://doi.org/10.24188/recia.v11.n1.2019.728

Universidad de Sucre, Colombia

Los autores permiten a RECIA reimprimir el material publicado en él. En caso de que un autor quiera traducir o usar una publicación parcial o completa de nuestro Diario, el autor debe obtener un permiso por escrito del editor de la revista.

Revista Colombiana de Ciencia Animal - RECIA está distribuido bajo una Licencia Creative Commons Atribución-CompartirIgual 4.0 Internacional. 


\title{
Descripción radiológica de lesiones en el tarso de caballos criollos colombianos
}

\section{Radiological description of lesions in the tarsus of colombian creole horses}

Renso Sneider Gallego Rodríguez. M.Sc.

DOI: https://doi.org/10.24188/recia.v11.n2.2019.728

Corporación Universitaria Remington, Facultad de Medicina

Veterinaria, Grupo de Investigación en Veterinaria (GINVER),

Medellín, Colombia.

renso.gallego@uniremington.edu.co

(iD) https://orcid.org/0000-0003-1563-9731

\section{Juan Diego Lujan Giraldo. MV}

Corporación Universitaria Remington, Facultad de Medicina Veterinaria, Grupo de Investigación en Veterinaria (GINVER), Medellín, Colombia.

diego-322@hotmail.com

D https://orcid.org/0000-0002-0590-7328

\section{Jesika Leysner Tavera. MV}

Corporación Universitaria Remington, Facultad de Medicina Veterinaria, Grupo de Investigación en Veterinaria (GINVER), Medellín, Colombia.

jesikaleysner@gmail.com

(i) https://orcid.org/0000-0003-2729-4195

Recepción: 30 Julio 2019

Aprobación: 9 Noviembre 2019

Publicación: 12 Noviembre 2019

\section{RESUMEN}

Las patologías articulares son uno de los eventos más frecuentes de claudicación en equinos, estas pueden ser causadas por diversos factores entre los que se incluye traumatismos recurrentes, inestabilidad articular, alteraciones de la osificación e infecciones. Los eventos inflamatorios articulares propician lesiones que llevarán a un deterioro del cartílago articular favoreciendo la enfermedad articular y la presentación de hallazgos clínicos. El presente es un estudio prospectivo de carácter descriptivo en el cual se realizaron y analizaron un total de 22 radiografías de tarso en equinos, se incluyeron un total de 11 caballos de características físicas homogéneas, a los cuales se les hizo un estudio radiológico de tarso bilateral para cada animal; en el análisis radiológico se evaluó la frecuencia de hallazgos radiográficos patológicos tales como: artrofitos, disminución del espacio articular, proliferación ósea, lisis ósea, entre otros, a su vez, se determinó del ángulo tarsal para cada una de las radiografías. Del total de radiografías se encontró que el 90.9\% presentó algún hallazgo radiológico patológico, a su vez, los artrófitos fueron las lesiones encontradas con mayor frecuencia en el estudio y los huesos tarso central, tarso tres las estructuras anatómicas más afectadas.

Palabras claves: Angulo tarsal, diagnóstico, equinos, radiografía, tarso.

\begin{abstract}
Joint pathologies are one of the most frequent causes of claudication in horses, these can be caused by several factors including recurring trauma, joint instability, ossification alterations and infections. Articular inflammatory events cause injuries that will lead to deterioration of the articular cartilage favoring joint disease and the presentation of clinical findings. The present is a prospective descriptive study in which a total of 22 tarsus x-rays were performed and analyzed in equines, a total of 11 horses of homogeneous physical characteristics were included, to which a radiological study
\end{abstract}


of bilateral tarsus was made for each animal; In the radiological analysis, the frequency of pathological radiographic findings was evaluated, such as: arthrophytes, decreased joint space, bone proliferation, bone lysis, among others, in turn, the tarsal angle was determined for each of the radiographs. Of the total number of radiographs, $90.9 \%$ showed some pathological radiological findings, in turn, the arthrophytes were the most frequently found lesions in the study and the central tarsal and tarsal bones were the three most affected anatomical structures.

Keywords: Tarsal angle, diagnosis, equines, radiography, tarsus.

\section{INTRODUCCIÓN}

Las articulaciones juegan un papel muy importante en el desplazamiento del animal manteniendo una estructura e integridad adecuada de sus extremidades y proporcionando un soporte adecuado. (1). La enfermedad articular es la causa más común de cojeras en equinos, se relaciona con factores como traumatismo constante, inestabilidad articular, infecciones y alteraciones de la osificación endocondral (2). Las articulaciones con más afección son las articulaciones del carpo, del tarso e interfalángicas, las cuales soportan mayor peso y presentan mayor movilidad en el equino (3).

Los factores pro inflamatorios que comúnmente afectan las articulaciones son: el factor de necrosis tumoral (TNF $\alpha$ ) y la interleucina 1 (IL-1) como los dos principales mediadores de la respuesta inflamatoria, también participan otras interleucinas, factores de necrosis, entre otros. Se ha descrito que las reacciones enzimáticas que se presentan en la enfermedad articular son mediadas por la activación de la proteína C reactiva como parte del sistema del complemento (4). Se menciona la acción de proteínas líticas tales como las colagenasa, las gelatinisas y la estromelisina como agentes degradadores del cartílago articular; a su vez se relaciona la acción de prostaglandinas, leucotrienos y óxido nítrico en la lesión articular (5).

Los métodos diagnósticos relacionados con la enfermedad articular abarcan desde el examen físico, goniometría articular, evaluación del líquido sinovial, técnicas imagenológicas, entre otros; el análisis del líquido sinovial se aborda desde el aspecto físico donde se evalúa la viscosidad, la turbidez, el color; a nivel del análisis celular, se tiene en cuenta la reacción leucocitaria, las proteínas plasmáticas y el aspecto microbiológico, en el cual mediante la técnica de cultivo se evidencia el crecimiento bacteriológico (6).

La radiografía es la técnica diagnóstica más utilizada en la evaluación de los cambios estructurales del cartílago articular, su relevancia se da al caracterizar los cambios patológicos del hueso en un estadio inicial-agudo o crónico, se menciona que hay un pobre correlación entre los signos clínicos y los hallazgos radiográficos presentes en animales con enfermedad articular, esto se sustentan en la hipótesis la cual menciona que los cambios radiográficos no están presentes en etapas tempranas de la patología (degradación del cartílago) lo cual llevaría al retraso en el diagnóstico y el tratamiento, afectando el pronóstico atlético del paciente (7). La ultrasonografía y la resonancia magnética, hacen parte de las técnicas imagenológicas que pueden ser empleadas con el fin de asociar hallazgos del tejido blando, tejido articular y estructura ósea del animal (8).

\section{MATERIALES Y MÉTODOS}

Aspectos éticos. El estudio se realizó bajo la normativa de la Ley No. 1774 del 6 de enero del 2016, en el cual se aseguró que ninguno de los animales presentes en el estudio tuviera algún signo de disconfort, fuera utilizado con fines experimentales o fuera vulnerado el reglamento de bienestar animal.

Unidad de Análisis. El estudio se realizó con 11 caballos criollos colombianos, entre los cuales había machos y hembras, con una edad comprendida entre los 4 - 12 años, con una alzada entre 1.45 y 1.55 metros, el peso se relacionaba de 300 - $380 \mathrm{~kg}$ de peso, ubicados en dos fincas diferentes, los cuales tenían como fin zootécnico la monta y trabajos de finca relacionados a carga de pasto, entre otros. A cada uno de los equinos se le hizo evaluación radiográfica de los tarsos teniendo como vistas principales la proyección antero - posterior y latero - medial de tarso, por lo tanto, se cuenta con 22 tarsos evaluados durante el estudio. En la selección de las unidades de análisis no se relacionó con la presentación de claudicaciones históricas por parte de los animales.

Evaluación Radiográfica. El estudio radiológico se realizó con un equipo generador VR40 VET_RAY INC, USA, Idaho 1989, la técnica con la que se realizaron las radiografías fue de 80 de kilovoltaje, 0,32 de miliamperaje y 0,32 miliamperaje/segundo; a partir de las dos vistas radiográficas (lateromedial de tarso y anteroposterior de tarso) 
se evaluaron las siguientes estructuras anatómicas: articulación tarso - crural, articulación intertarsiana proximal, articulación intertarsiana distal, articulación tarso - metatarsiana, articulación talo - calcanea. Se realizó evaluación del ángulo tarsiano teniendo en cuenta lo descrito por Galisteo et al. 1996; Cano et al. 1999, quienes mencionaron que para determinar el ángulo tarsal se debía identificar el punto de intersección, comprendido entre el espacio del cuarto hueso tarsiano, cuarto hueso metatarsiano y el tercer hueso metatarsiano; este ángulo fue medido en cada estudio radiológico; según lo anterior se tuvo como valor normal $148,42 \pm 2,65^{\circ}$.

Sistema de Evaluación. El análisis radiológico se hizo por dos médicos veterinarios especialistas en diagnóstico por imagen y en medicina interna de equinos. Como variable inicial se realizó la medición del ángulo articular del tarso, dicha medición se hizo a nivel radiográfico teniendo como puntos específicos el eje medio del talus. Para realizar una evaluación diagnóstica de los hallazgos obtenidos a nivel del tarso del caballo mediante la radiología se tuvo en cuenta la presencia o ausencia de los siguientes hallazgos: proliferación ósea, reacción perióstica, disminución del espacio articular, lisis ósea, luxación articular, esclerosis, osteofitos, artrofitos, entesofitos, fracturas y anquilosis articular; a su vez, se describieron las estructuras anatómicas más afectadas.

Evaluación del Tarso. Se realizó la evaluación de la articulación tarsocrural con el fin de evidenciar disminución del espacio articular, proliferación ósea o fragmentación del cartílago articular (osteofitos). Las articulaciones distales tarsales fueron evaluadas para la presencia de artrofitos, lisis, esclerosis, disminución del espacio articular y fracturas óseas. Todos los hallazgos fueron descritos según el hueso o la estructura anatómica afectada.

Tipo de estudio y descripción estadística. Se realizó un estudio retrospectivo en el cual se analizaron las radiografías de tarso equino presentes en la clínica veterinaria de la Universidad Remington - Medellín Colombia. Se hicieron medidas de estadística descriptiva en la cual se evaluó la frecuencia de la presentación de hallazgos y lesiones a nivel radiológico por medio de una base de datos categorizada en Excel.

\section{RESULTADOS}

La cantidad de tarsos con lesiones en el estudio radiográfico se tabulan en la tabla 1, donde se encontró que, de los 22 tarsos evaluados, 20 presentaban algún tipo de lesión evaluable a nivel radiológico (Tabla 1). De las estructuras anatómicas analizadas mediante las radiografías se evidenció que las articulaciones intertarsianas distales, el tarso 3 y el tarso central fueron las más afectadas y donde se presentaron mayor cantidad de lesiones.

Tabla 1. Evaluación de hallazgos radiológicos del tarso en equinos

\begin{tabular}{ccc}
\hline Examen Radiológico & Radiografía Tarsos $(\mathrm{n}=22)$ & $\%$ \\
\hline Hallazgos Patológicos & 20 & 90,9 \\
Sin Hallazgos & 2 & 9.09 \\
\hline
\end{tabular}

Se realizó el análisis y la descripción de las lesiones a nivel radiológico (Figura 1), se debe aclarar que fueron encontrados varias lesiones en la evaluación de cada una de las unidades de análisis. De los hallazgos obtenidos se obtuvo que los más relevantes fueron la presentación de artrofitos, los cuales representaron la mayor cantidad con un porcentaje de 86.4\%, seguido de la disminución del espacio articular con un 59.1\%, a su vez, la proliferación ósea y la lisis ósea tuvieron un $13.6 \%$ cada uno (Tabla 2 ).

Tabla 2. Presentación de hallazgos radiológicos encontrados en el tarso

\begin{tabular}{ccc}
\hline Hallazgo Radiológico Identificados & $\mathbf{S i}$ & $\mathbf{\%}$ \\
\hline Artrofito & 19 & 86.4 \\
Disminución del espacio articular & 13 & 59.1 \\
Proliferación ósea & 3 & 13.6 \\
Lisis ósea & 3 & 13.6 \\
Reacción Perióstica & 2 & 9.1 \\
Esclerosis & 2 & 9.1 \\
Osteofito & 1 & 4.5 \\
Luxación & 0 & 0.0 \\
\hline
\end{tabular}




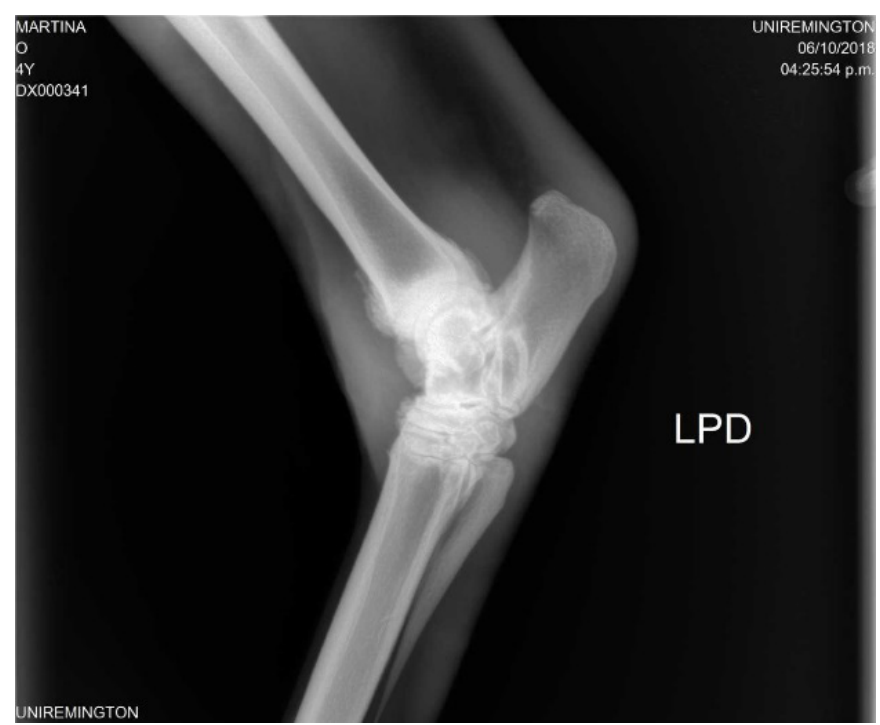

Figura 1. Proyección radiográfica de tarso lateral, en la cual se observa reacción perióstica moderada a nivel del aspecto anterior de la porción distal de la tibia, lisis ósea en la tróclea del talus y artrofitos con disminución del espacio de la articulación intertarsiana proximal.

Según los datos obtenidos en las mediciones realizadas para establecer los ángulos tarsales (goniometría) se encontró que el 68.18\% (15 animales) presentaron un aumento del ángulo normal establecido, así mismo, el 18.18\% (4 animales) tuvieron un ángulo disminuido, y sólo el 13.6\% (3 animales) se encontró con ángulo normal del tarso.

\section{DISCUSIÓN}

Autores como Roger et al (9) en el 2012, aseguran que la enfermedad musculoesquelética es la principal causa de retiro de los caballos deportistas (9). En un estudio en el cual se realizaron radiografías articulares con el fin de evidenciar la patología más común, encontraron que la osteoartritis fue el hallazgo más común con el $94.6 \%$ de los casos (10). Lo mencionado anteriormente se asocia con los resultados obtenidos ya que hallazgos radiográficos como artrofitos, disminución del espacio articular, proliferación ósea, lisis ósea, entre otros, se relacionan con eventos inflamatorios articulares que a nivel clínico son relacionados con el diagnóstico de osteoartritis en equinos.

Las radiografías de tarso se hacen de forma rutinaria, especialmente en motivos de consulta que están relacionados con disminución del rendimiento, examen precompra y claudicación (11), autores como Jackman en el 2001, mencionan que de un estudio en el cual se evaluó radiográficamente 22 caballos encontraron que $69.4 \%$ presentaron cambios o lesiones en el tarso (12). Los datos mencionados anteriormente presentan relación con lo encontrado en el presente estudio, ya que a pesar de que solo se tomaron radiografías del tarso, el porcentaje de lesiones encontradas fue alto con un $90.9 \%$ del total de 22 radiografías realizadas.

Una de las patologías que más se encuentra a nivel del tarso es la osteoartritis, según Jacklin Ben (13) en el 2016, la articulación tarsometatarsiana y las intertarsianas distales son las más afectadas y son un motivo común de cojera en el paciente equino (13). Lo cual esta relacionado con lo encontrado en este estudio, ya que la mayor evidencia de hallazgos radiológicos y lesiones se encontraron a nivel de la articulación intertarsiana distal, así mismo, el tarso central y el tarso 3 fueron los más afectados.

En las mediciones del ángulo tarsal descritas por Llorca M.C. (14) en el 2016, se menciona una mediana de $149.58^{\circ}$ con valores que van desde $143.47^{\circ}$ hasta $154.57^{\circ}$, el autor menciona que en los datos obtenidos hubo correlación significativa debido a la homogeneidad de la población analizada ya que se trataba de individuos de característica similares pertenecientes a una misma raza (14). A diferencia de lo mencionado anteriormente, los valores del ángulo tarsal obtenidos en el presente estudio fueron heterogéneos ya que un alto porcentaje de la población tuvo valores superiores relacionados al rango normal $\left(148.42 \pm 2.65^{\circ}\right.$.), esto puede relacionarse con el hecho de que casi todos los animales presentes evidenciaron algún tipo de lesión a nivel radiológico por lo tanto se mencionan como clínicamente afectados. Autores como Barcelos et al (15) en el 2016, mencionaron realizaron un estudio en el cual se encontró una mayor prevalencia de patologías en caballos con un ángulo tarsal mayor o igual a $140^{\circ}(15)$. 
Diversos estudios han investigado acerca de los ángulos tarsales normales, literatura anterior menciona que el rango normal del ángulo tarsal es de $140^{\circ}-160^{\circ}$ siendo mayor para caballos de carrera y menor para caballos de tiro (16). Estudios posteriores mostraron resultados similares encontrando que el ángulo normal para caballos de carrera se encontró entre $155^{\circ}$ y $160^{\circ}$ (17). En estudios más actuales se Gnagey et al (18) en el 2006, menciona que el valor normal de los ángulos tarsales es de $155.5^{\circ}-165.5^{\circ}$ (18). Según las referencias mencionadas anteriormente se debe tener en cuenta que son varias las adaptaciones descritas para los ángulos tarsales normales en el equino y que esto depende del tamaño y de la función deportiva del animal.

En conclusión la presentación de hallazgos radiológicos patológicos a nivel del tarso en el equino es un evento común, de los cuales el artrofito se presenta con mayor frecuencia afectando comúnmente el hueso tarso central y el hueso tarso tres; la disminución del espacio articular fue otro de los hallazgos encontrados con mayor frecuencia afectando la articulación tarsotibial y la intertarsiana proximal.

\section{Conflicto de intereses}

No hay ningún conflicto de interés en cuanto a los datos mencionados y/o publicados en este documento.

\section{REFERENCIAS}

1. Rolando J. Aplicación de la determinación de las metaloproteinasas de la matriz (MMP) en las enfermedades articulares equinas. Fisiología animal y laboratorio del metabolismo óseo 2013. https://bit.ly/36rDNlS

2. Weeren R. Joint physiology: responses to exercise and training. In: Equine Sports Medicine \& surgery. Edinburgh: Elsevier; 2014. https://www.amazon.es/Equine-Sports-Medicine-Surgery-clinical/dp/0702047716

3. Sommer NH, Carvalho B, Galecio S, Paredes E, Gómez M. Avances en Osteoartritis equina. Universidad Austral de Chile: Chile; 2012. http://cybertesis.uach.cl/tesis/uach/2012/fvs711a/doc/fvs711a.pdf

4. Polli M, Caggiano N, Perrone G, Marino M, De Simone E, Chaipe. A. Variación del nivel de citoquinas en líquido sinovial de equinos con enfermedad articular tratados con biofosfonatos. Rev Complut Ciencias Vet. 2013; 7(1):69-83. https://doi.org/10.5209/rev RCCV.2013.v7.n1.42064

5. Sandoval JA, López C, Carmona JU. Therapies intended for joint regeneration in the horse. Arch Med Vet. 2013; 45(3):229-36. http://dx.doi.org/10.4067/S0301-732X2013000300002

6. Steel, C. Equine Synovial Fluid Analysis. Vet Clin Equine Pract. 2008; 24(2):437-54. https://doi.org/10.1016/j. cveq.2008.05.004

7. Jara E, Corrêa F. Osteoartritis en equinos : descripción , diagnóstico y alternativas terapéuticas. Food Environ Res. 2016; 4(3):16-39. DOI: https://doi.org/10.7770/safer-V4N3-art1041

8. Caron J. Arthritis: Osteoarthritis. In: Diagnosis and Management of Lameness in the Horse. Saunder Elsevier. 2011; 655-68. https://doi.org/10.1016/C2009-0-50774-X

9. Rogers CW, Bolwell CF, Tanner JC WVR. Early exercise in the horse. J Vet Behav. 2012; 7(6):375-379. Doi: https:// doi.org/10.1016/j.jveb.2012.01.003

10. Garcia RS, Melo UP, Ferreira C, Toskano FS CG. Estudo clinico e radiográfico da osteoartrite tarsica juvenil em potros da raça mangalarga marchador. Ci Anim Bras. 2009; 10(1):254-60. https://www.revistas.ufg.br/vet/article/ view/2316

11. Judy CE, Acvs D. Radiography of the Carpus and Hock. 2013; 59:372-8. https://aaep.org/sites/default/files/ issues/RadiographJudy.pdf 
12. Jackman BR. Common lamenesses in the cutting and reining horse. Proc Am Ass Equine Pr. 2001; 47:6-11. https:// pdfs.semanticscholar.org/503b/a147b6e918253fef4dadc2b7446ce588cdce.pdf

13. Jacklin B. Radiology of the equine hock and proximal cannon. In Pract. 2016; 38(8):399-407. http://dx.doi. org/10.1136/inp.i4106

14. Carlos M, Miravet L. Influencia del ángulo tarsal en el esparaván del caballo pre de tres años. [Tesis Ph.D.]. Universidad de Zaragoza; España; 2016; https://zaguan.unizar.es/record/48121/files/TESIS-2016-081.pdf.

15. Da Costa Barcelos KM, de Rezende ASC, Biggi M, Lana ÂMQ, Maruch S, Faleiros RR. Prevalence of Tarsal Diseases in Champion Mangalarga Marchador Horses in the Marcha Picada Modality and Its Association With Tarsal Angle. J Equine Vet Sci. 2016; 47:25-30. https://doi.org/10.1016/i.jevs.2016.07.012

16. Jardim WR. Exterior e Julgamento dos Equídeos. Piracicaba: Tip Aloisi. 1940. https://books.google.com.co/boo

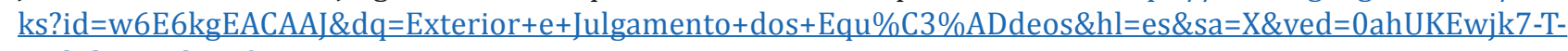
ot3lAhVRx1kKHfpcAnAQ6AEIKDAA

17. Chieffi A, Camargo MX. Ezoognósia: exterior dos grandes animais domésticos In: São Paulo: Edanne. 1971. p. 188. https://books.google.com.co/books?id=ZdS6XwAACAAJ\&dq=Ezoogn\%C3\%B3sia:+exterior+dos+grandes+ani$\underline{\text { mais+dom\%C3\%A9sticos\&hl=es\&sa=X\&ved=0ahUKEwiH0p20093lAhVFjlkKHZ6BDkYQ6AEIKzAA }}$

18. Gnagey L, Clayton HM, Lanovaz JL. Effect of standing tarsal angle on joint kinematics and kinetics. Equine Vet J. 2006;38:628-33. DOI: https://doi.org/10.2746/042516406X159043 